\title{
Astragaloside IV alleviates the symptoms of experimental ulcerative colitis in vitro and in vivo
}

\author{
SUXIAO WU and ZILAN CHEN \\ Department of Gastroenterology, Suzhou Municipal Integrated Traditional Chinese and Western Medicine Hospital, \\ Suzhou, Jiangsu 215101, P.R. China
}

Received December 10, 2018; Accepted July 19, 2019

DOI: $10.3892 /$ etm.2019.7907

\begin{abstract}
Ulcerative colitis (UC) is a chronic and relapsing inflammatory intestinal disease. Although the morbidity of $\mathrm{UC}$ has increased notably in recent years, effective therapeutic treatment remains unsatisfactory. Astragaloside IV (ASI), a monomeric compound isolated from the traditional Chinese medicine herb Ligusticum chuanxiong, exhibits anti-inflammatory effects. The present study aimed to investigate the therapeutic effects of ASI on experimental UC in vitro and in vivo. Cell proliferation was detected via a Cell Counting Kit-8 assay in vitro. In addition, the concentrations of the inflammatory factors myeloperoxidase (MPO), tumor necrosis factor- $\alpha$ (TNF- $\alpha$ ), interleukin-1 $\beta$ (IL-1 $\beta$ ), interleukin-6 (IL-6) and nitric oxide (NO) in the colon tissues were determined by ELISA. Western blot analysis was used to examine phosphorylated transcription factor p65 (p-p65), p-inhibitor of NF- $\mathrm{NB}(\mathrm{I} \kappa \mathrm{B})$, claudin-1 and tight junction protein $\mathrm{ZO}-1$ (ZO-1) protein levels in vitro and in vivo, respectively. The results indicated that lipopolysaccharide (LPS) significantly increased the pro-inflammatory cytokines TNF- $\alpha$, IL-1 $\beta$ and IL-6 in CCD-18Co cells, which was markedly ameliorated by ASI. In addition to the inhibition of pro-inflammatory cytokines, ASI decreased the levels of p-p65 and p-IкB proteins. In addition, ASI decreased the disease activity index scores, and increased colon lengths in dextran sulfate sodium-induced UC mice. ASI also decreased the levels of the pro-inflammatory factors MPO, TNF- $\alpha$, IL-1 $\beta$, IL-6 and NO, and upregulated the expression of claudin-1 and ZO-1 in colon tissues. Therefore, ASI was effective in ameliorating experimental UC in vitro and in vivo via the inhibition of inflammatory molecules, and the downregulation of $\mathrm{NF}-\kappa \mathrm{B}$ signaling. In conclusion, ASI
\end{abstract}

Correspondence to: Dr Zilan Chen, Department of Gastroenterology, Suzhou Municipal Integrated Traditional Chinese and Western Medicine Hospital, 39 Xiasha Tang, Mudu, Wuzhong, Suzhou, Jiangsu 215101, P.R. China

E-mail: zilian_chen@126.com

Key words: astragaloside IV, ulcerative colitis, inflammatory molecules, lipopolysaccharide, dextran sulfate sodium may serve as a potential therapeutic agent for the treatment of UC.

\section{Introduction}

Ulcerative colitis (UC) is a chronic and relapsing inflammatory intestinal disease, which is characterized by acute pain, diarrhea and weight loss $(1,2)$. Despite progress in previous decades, the etiology and pathogenesis of ulcerative colitis remain unclear (3). Multiple factors, including dysbiosis of commensal microbiota, dysfunction of the mucosal barrier and a defective immune response, may contribute to the etiology of UC $(4,5)$. At present, the morbidity of UC is increasing annually (6). Currently, aminosalicylic acid preparations, glucocorticoids and immunosuppressants are the three major classes of drugs for the clinical treatment of UC (7). However, the side effects and sometimes serious adverse reactions to these drugs greatly limit their use in the treatment of $\operatorname{UC}(7,8)$. Therefore, more effective therapeutic strategies for treating UC are required.

Plant-derived natural products exhibit distinctive chemical diversity (9). Previous studies have indicated that medicinal plant-derived extracts including polysaccharides and flavonoids have anti-UC activity, by regulating the levels of inflammatory mediators $(10,11)$.

Astragaloside IV (ASI) is a monomeric compound isolated from the traditional Chinese herb Astragalus membranaceus (12). Previous studies indicated that ASI possesses extensive pharmacological activities, including regulation of intestinal microbiota, and anti-tumor and anti-inflammatory activities (13-15). A previous study indicated that ASI may alleviate heat-induced inflammation by regulating endoplasmic reticulum stress (15). ASI also improves renal fibrosis through the inhibition of the NF- $\mathrm{B}$ signaling pathway (16). Kang et al (17) indicated that Wasabia japonica prevented $\mathrm{UC}$ via inhibiting the NF- $\kappa \mathrm{B}$ signaling pathway. In addition, ASI may attenuate lipopolysaccharide (LPS)-induced cardiac dysfunction and inflammation by inhibiting the levels of tumor necrosis factor- $\alpha$ (TNF- $\alpha$ ), interleukin (IL)-1 $\beta$ and IL-6 in mice (18). Jiang et al (19) identified that ASI alleviated colonic mucosal injury in a mouse model of colitis (19). However, the specific effect of ASI on experimental UC in mice remains unclear. Therefore, the present study aimed to explore the role of ASI in experimental UC in vivo and in vitro. 


\section{Materials and methods}

Cell line and cell culture. Human colon fibroblast CCD-18Co cells were purchased from The American Type Culture Collection. Cells were cultured in Dulbecco's modified Eagle's medium (Thermo Fisher Scientific, Inc.) supplemented with 10\% FBS (Gibco; Thermo Fisher Scientific, Inc.), penicillin and streptomycin $(100 \mathrm{U} / \mathrm{ml})$ in a humidified $5 \% \mathrm{CO}_{2}$ incubator at $37^{\circ} \mathrm{C}$.

Cell viability detection. A Cell Counting Kit-8 (CCK-8; Beyotime Institute of Biotechnology) assay was used to evaluate the cell viability, according to the manufacturer's protocols. CCD-18Co cells were seeded into a 96-well plate $\left(5 \times 10^{3}\right.$ cells/well) and cultured overnight. Then, CCD-18Co cells were pretreated with ASI $(0,10$ or $50 \mu \mathrm{M})$ for $24 \mathrm{~h}$, followed by treatment with LPS (0 or $1 \mu \mathrm{g} / \mathrm{ml})$ for an additional $2 \mathrm{~h}$. After $24 \mathrm{~h}$ incubation, $10 \mu \mathrm{l}$ CCK- 8 reagent was added to each well. The absorbance values were measured at a wavelength of $450 \mathrm{~nm}$ using a microplate reader (Bio-Rad Laboratories, Inc.). LPS was provided by Sigma-Aldrich; Merck KGaA. ASI was purchased from MedChemExpress.

ELISA for detection of pro-inflammatory factors. CCD-18Cocells were seeded into a 24 -well plate $\left(2 \times 10^{6}\right.$ cells/well $)$ and cultured overnight. Cells were pretreated with ASI $(0,10$ or $50 \mu \mathrm{M})$ for $24 \mathrm{~h}$, followed by treatment with LPS $(0$ or $1 \mu \mathrm{g} / \mathrm{ml})$ for an additional $2 \mathrm{~h}$. Then, after $24 \mathrm{~h}$ incubation, samples of the supernatant were collected from each well to measure TNF- $\alpha$, IL-1 $\beta$ and IL-6 levels by ELISA. TNF- $\alpha$ (cat. no. H052), IL-1 $\beta$ (cat. no. H002) and IL-6 (cat. no. H007) ELISA kits were purchased from Nanjing Jiancheng Bioengineering Institute.

Western blot analysis. CCD-18Co cells and segments of mouse colon were collected and homogenized using radioimmunoprecipitation assay lysis buffer (Thermo Fisher Scientific, Inc.) supplemented with protease and phosphatase inhibitors. A Bradford protein assay (Beyotime Institute of Biotechnology) was used to quantify the protein concentration. Equal amounts of proteins $(30 \mu \mathrm{g})$ in the lysate were then separated via 10\% SDS-PAGE and transferred onto a polyvinylidene fluoride (PVDF) membrane (Thermo Fisher Scientific, Inc.) for $2 \mathrm{~h}$. The PVDF membranes were blocked in TBST containing 5\% skim milk at room temperature for $1 \mathrm{~h}$, and then incubated at $4^{\circ} \mathrm{C}$ with primary antibodies overnight. The primary antibodies were as follows: Rabbit anti-NF- $\mathrm{KB}$ transcription factor p65 (p65; 1:1,000; cat. no. ab16502), rabbit anti-phosphorylated NF- $\kappa \mathrm{B}$ transcription factor p65 (p-p65; 1:1,000; cat. no. ab86299), rabbit anti-inhibitor of NF- $\mathrm{KB}$ (IкB; 1:1,000; cat. no. ab32518), rabbit anti-phosphorylated inhibitor of NF-кB (p-ІкB; 1:1,000; cat. no. ab133462), rabbit anti-claudin-1 (1:1,000; cat. no. ab15098), rabbit anti-tight junction protein ZO-1 (ZO-1; 1:1,000; cat. no. ab96587) and anti- $\beta$-actin (1:1,000; cat. no. ab8227). The PVDF membranes were washed 3 times with TBST, then incubated with horseradish peroxidase-conjugated goat anti-rabbit IgG H\&L secondary antibody (1:5,000; cat. no. ab7090) at room temperature for $1 \mathrm{~h}$. All antibodies were purchased from Abcam. The protein blots were detected with an enhanced chemiluminescence system (Bio-Rad Laboratories, Inc.) and visualized using a Fluor Chem E imager on a FluorChem E Imaging System 46688 (ProteinSimple). The density of the blots for target proteins were normalized to $\beta$-actin.

Dextran sulfate sodium (DSS)-induced ulcerative colitis in mice. Male C57BL/6 mice (21-23 g, aged 8-10 weeks) were provided by Shanghai SLAC Laboratory Animal Co., Ltd. The animals were housed at a temperature of $24 \pm 1^{\circ} \mathrm{C}$, a $12 \mathrm{~h}$ light: Dark schedule starting at 08:00, and a humidity of $50-60 \%$ for 4 days prior to the initiation of the experiment. The animals were provided with water and food ad libitum.

Following acclimation, the 30 mice were randomly divided into five experimental groups ( $\mathrm{n}=6)$ : Control (water); model group (DSS); DSS $+50 \mathrm{mg} / \mathrm{kg}$ ASI (DSS/ASI); DSS + $200 \mathrm{mg} / \mathrm{kg}$ ASI (DSS/ASI); and $200 \mathrm{mg} / \mathrm{kg}$ ASI treatment (ASI). The mice were orally administered 50 or $200 \mathrm{mg} / \mathrm{kg}$ ASI once daily for 3 days (from day 0 to day 3). On day 3, UC was induced in the animals via oral administration of $3 \%(\mathrm{w} / \mathrm{v})$ DSS (ad libitum; MP Biomedicals) in fresh drinking water for 5 days. From day 8 , animals were orally administered 50 or $200 \mathrm{mg} / \mathrm{kg}$ ASI alone until the end of the experiment. The body weight of the mice was monitored every other day. The disease activity index (DAI) score was determined as described previously (20). At the end of the experiments, the mice were sacrificed using $\mathrm{CO}_{2}$ at a displacement rate of $20 \%$ of the chamber volume/min $\left(\mathrm{CO}_{2}\right.$ flow rate, $\left.2.5 \mathrm{l} / \mathrm{min}\right)$. The length of the colon between the ileocecal junction and the proximal rectum in the mice was measured. The colon tissues were immediately fixed in $4 \%$ formalin overnight at room temperature, embedded in paraffin wax for histological analysis and stored at $-80^{\circ} \mathrm{C}$ for other experiments. All experimental procedures were approved by the Ethical Committee of Suzhou Integrated Traditional Chinese and Western Medicine Hospital. The National Institutes of Health Guide for the Care and Use of laboratory animals was followed (21).

Histological evaluation. Colon specimens from the different groups were embedded in paraffin and fixed with $4 \%$ formalin at room temperature for 2 weeks, and sliced at a 5 -mm thickness. These fixed tissues were stained via hematoxylin-eosin staining, as described previously (22). A BH22 light microscope (Olympus Corporation; magnification, $\mathrm{x} 400$ ) was used to observe the histological damage and inflammation of the colon tissues. Severity of histological inflammation was graded as described previously $(23,24)$ : Ulceration $(0$, none; 1 , erosion; 2 , submucosa ulceration); crypt abscesses ( 0 , none; 1 , mild; 2 , severe), degree of mononuclear cell infiltration (MNCI) (0, no infiltration; $1,<25 \% ; 2,25-50 \% ; 3,50-75 \%$; $4,>75 \%$ ); segmental distribution of MNCI ( 0 , continuous; 1 , mildly segmental; 2 , markedly segmental); and eosinophil infiltration ( 0 , none or minimal; 1 , mild; 2 , severe).

Nitric oxide $(\mathrm{NO})$ detection. The colon tissues were freeze-thawed in liquid nitrogen and levigated. The NO concentration in the colon tissues was detected by Griess assay (Sigma Aldrich; Merck KGaA) as previously described (2). The data are presented as the mean (nitrite) in $\mathrm{mM} / \mathrm{g}$ colon tissue.

Measurement of cytokines by ELISA in vivo. The TNF- $\alpha$ (cat. no. H052), IL-1 $\beta$ (cat. no. H002) and IL-6 (cat. no. H007) 

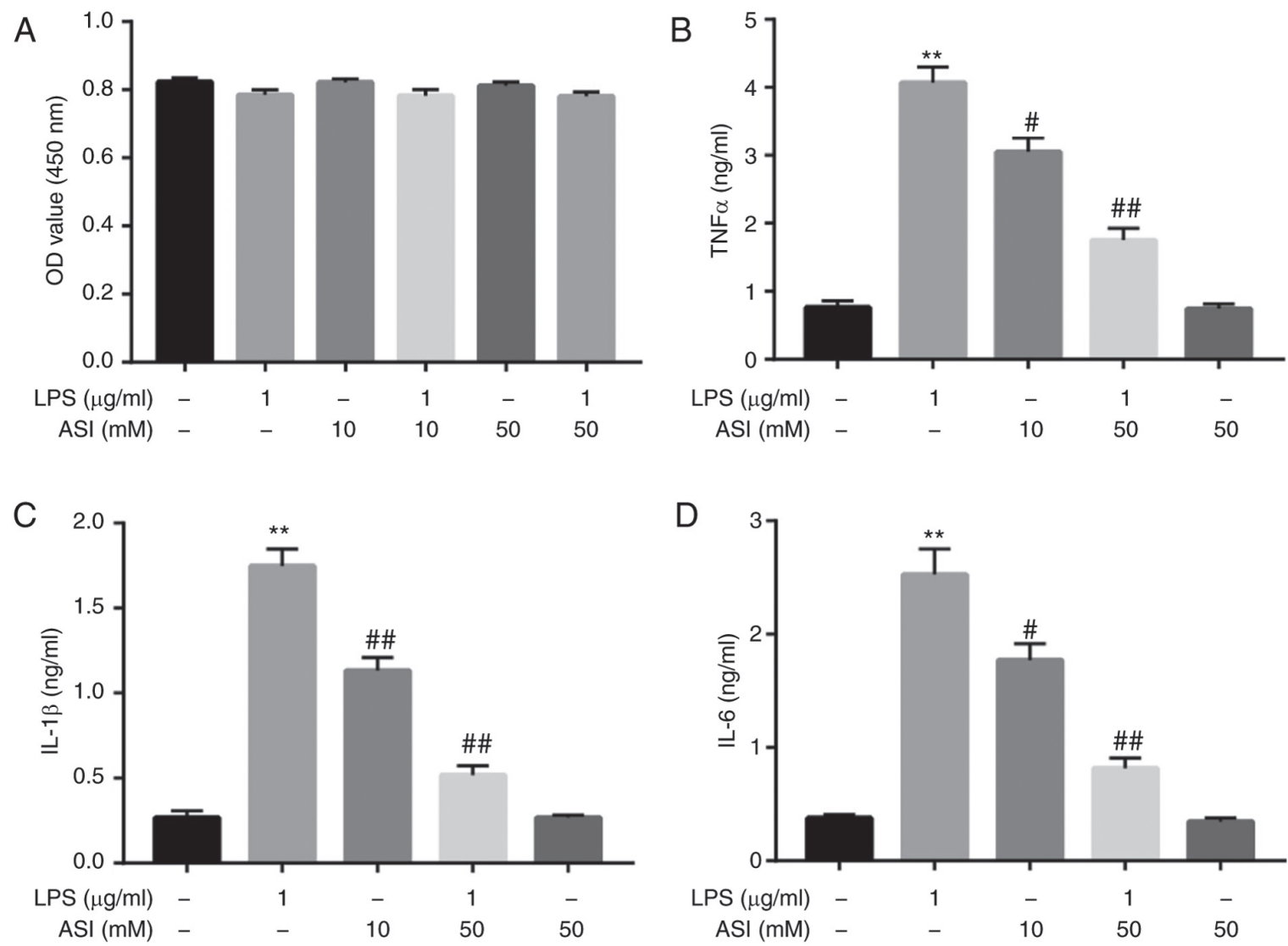

Figure 1. ASI decreases the production of TNF- $\alpha$, IL-1 $\beta$ and IL-6 in LPS-treated CCD-18Co cells in vitro. CCD-18Co cells were pretreated with ASI $(0,10$, or $50 \mu \mathrm{M})$ for $24 \mathrm{~h}$, following treated LPS $(1 \mu \mathrm{g} / \mathrm{ml})$ for $2 \mathrm{~h}$. (A) The viability of CCD-18Co cells was examined using a CCK-8 assay. (B) The level of TNF- $\alpha$ in the culture media was measured with ELISA. (C) The level of IL-1 $\beta$ in the culture media was measured with ELISA. (D) The level of IL-6 in the culture media was measured. ${ }^{* *} \mathrm{P}<0.01$ vs. the control group. ${ }^{~} \mathrm{P}<0.05$ and ${ }^{\# \#} \mathrm{P}<0.01$ vs. the LPS-treated group. ASI, astragaloside IV; TNF- $\alpha$, tumor necrosis factor- $\alpha$; IL, interleukin; LPS, lipopolysaccharide.

content in the colon tissues of the mice was measured with ELISA kits (Nanjing Jiancheng Bioengineering Institute) according to the manufacturer's protocol.

Measurement of neutrophil infiltration. The infiltration of neutrophils in the colon samples was assessed via myeloperoxidase (MPO) activity. Colon tissues were first homogenized in PBS (w/v 1/9), then the MPO activity assay kit (Nanjing Jiancheng Bioengineering Institute) was used to detect the MPO activity according to the manufacturer's protocol. The results are presented as $\mathrm{U} / \mathrm{g}$ wet tissue.

Statistical analysis. Each group was analyzed in at least three independent experiments and all data are presented as the mean \pm standard deviation. SPSS 17.0 software (SPSS, Inc.) was used for all statistical analyses. Comparisons among multiple groups were performed with one-way analysis of variance followed by Dunnett's post-hoc test. $\mathrm{P}<0.05$ was considered to indicate a statistically significant difference.

\section{Results}

ASI decreases the production of TNF- $\alpha, I L-\beta$ and IL- 6 in $L P S$-stimulated CCD-18Co cells in vitro. The potential cytotoxic effects of ASI and/or LPS on CCD-18Co cells were initially assessed using a CCK- 8 assay. As indicated in Fig. 1A, neither ASI nor LPS affected the cell viability of CCD-18Co cells. A previous study indicated that ASI exhibited gastro-protective effects against acute gastric lesions in rats by alleviating inflammation (25). The anti-inflammatory action of ASI in LPS-treated CCD-18Co cells was subsequently investigated. As demonstrated in Fig. 1B-D, compared with control treatment, LPS induced a significant increase in TNF- $\alpha$, IL- $\beta$ and IL-6 levels in cells. By contrast, LPS-induced TNF- $\alpha$, IL- $\beta$ and IL- 6 upregulation were significantly reversed by $50 \mu \mathrm{M}$ ASI. These data demonstrated that ASI may decrease the production of TNF- $\alpha$, IL- $\beta$ and IL- 6 in LPS-stimulated CCD-18Co cells in vitro.

ASI inhibits the phosphorylation of $N F-\kappa B$ p65 and $I \kappa B$ in LPS-treated CCD-18Co cells in vitro. It has been suggested that NF- $\mathrm{BB}$ serves as a key factor in the mediation of inflammation, and phosphorylated NF- $\kappa$ B p 65 (p-p65) serves a vital role in the activation of NF- $\mathrm{kB}(26)$. Therefore, the present study investigated whether ASI exerted anti-inflammatory effects on CCD-18Co cells via the inhibition of NF- $\kappa \mathrm{B}$ activation. As indicated in Fig. 2, the expression of p-p65 and p-IкB in cells was significantly increased by LPS. By contrast, the LPS-induced increases in $\mathrm{p}-\mathrm{p} 65$ and $\mathrm{p}-\mathrm{I} \kappa \mathrm{B}$ protein levels were notably reduced by $50 \mu \mathrm{M}$ ASI. These data demonstrated that ASI inhibited 

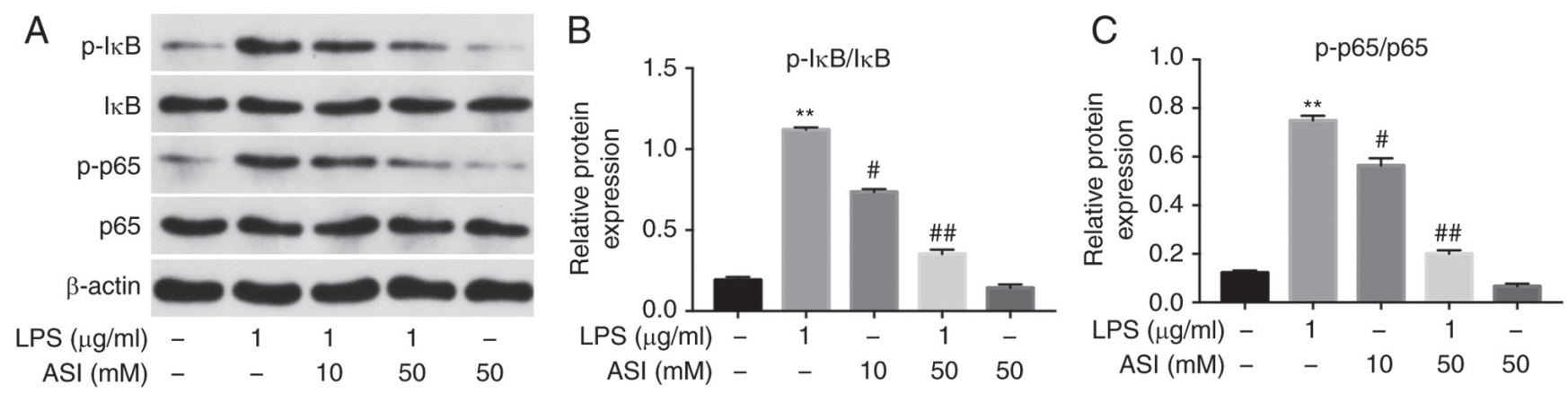

Figure 2. ASI inhibits phosphorylation of NF- $\kappa \mathrm{B}$ p65 and $\mathrm{I} \kappa \mathrm{B}$ in LPS-induced CCD-18Co cells in vitro. CCD-18Co cells were pretreated with ASI $(0,10$, or $50 \mu \mathrm{M})$ for $24 \mathrm{~h}$, followed by treatment with LPS $(1 \mu \mathrm{g} / \mathrm{ml})$ for $2 \mathrm{~h}$. (A) Expression levels of p-p65 and IкB in cells were investigated via western blot analysis. (B) The relative expression of $\mathrm{p}-\mathrm{I} \kappa \mathrm{B}$ was quantified via normalization to I $\mathrm{B}$. (C) The relative expression of $\mathrm{p}$-p65 was quantified via normalization to p65. ${ }^{* *} \mathrm{P}<0.01$ vs. the control group. ${ }^{\#} \mathrm{P}<0.05$ and ${ }^{\# \#} \mathrm{P}<0.01$ vs. the LPS treated group. ASI, astragaloside IV; LPS, lipopolysaccharide; $\mathrm{p}$, phosphorylated; p65, transcription factor $\mathrm{p} 65$; I $\mathrm{B}$, inhibitor of $\mathrm{NF}-\kappa \mathrm{B}$.

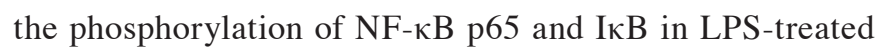
CCD-18Co cells in vitro.

ASI attenuates DSS-induced UC in mice. In order to confirm the anti-inflammatory effects of ASI in vivo, a DSS-induced mouse UC model was established. As indicated in Fig. 3A, compared with the control group, the body weights of the mice were markedly decreased in the DSS group. However, ASI $(200 \mathrm{mg} / \mathrm{kg})$ notably prevented body weight loss during the progression of experimental UC in mice (Fig. 3A). In addition, H\&E staining was used to observe the infiltration of inflammatory cells in sections of mouse colon segments. As indicated in Fig. 3B, colons in the control group were healthy, exhibiting intact surface epithelia and submucosa. However, DSS resulted in distortion of the crypt epithelium, and ulceration and damage to the surface epithelium. Compared with the DSS group, colons in the ASI $(200 \mathrm{mg} / \mathrm{kg})$ group exhibited well-preserved intact surface epithelia and cryptal glands (Fig. 3B). The histological damage scores in the ASI $(200 \mathrm{mg} / \mathrm{kg})$ group were significantly decreased compared with the DSS group (Fig. 3C). In addition, $200 \mathrm{mg} / \mathrm{kg}$ ASI significantly decreased the DAI during the progression of experimental UC in mice, which reflects the health status of the mice (Fig. 3D). Furthermore, ASI $(200 \mathrm{mg} / \mathrm{kg})$ significantly alleviated colon shortening caused by DSS in mice (Fig. 3E). All these data demonstrated that ASI attenuated UC induced by DSS in vivo.

ASI alleviates inflammatory responses in the colon in DSS-treated mice. Next, the concentrations of TNF- $\alpha$, IL-1 $\beta$ and IL-6 in colon tissues were detected in vivo. As indicated in Fig. 4A-C, the pro-inflammatory cytokines TNF- $\alpha$, IL-1 $\beta$ and IL-6 in the colon tissues were significantly upregulated in the DSS group, compared with the control group $(\mathrm{P}<0.01)$. However, ASI $(200 \mathrm{mg} / \mathrm{kg})$ treatment markedly suppressed the DSS-induced production of these cytokines (Fig. 4A-C). Similar to the pro-inflammatory cytokines, the results for NO and the MPO activity assay indicated that the levels of NO and MPO in the colon tissues were significantly upregulated in the DSS group. Nevertheless, ASI $(200 \mathrm{mg} / \mathrm{kg})$ treatment alleviated the DSS-induced production of NO and MPO in the colon (Fig. 4D and E). These data demonstrated that
ASI may alleviate inflammatory responses in the colon in the DSS-induced UC mouse model.

ASI increases the expression of tight junction (TJ) proteins in colonic tissues. It has been demonstrated that UC is associated with increased intestinal penetrability and decreased TJ protein expression, including that of ZO-1 and claudin-1 (27). As presented in Fig. 5, the expression levels of claudin-1 and ZO-1 were markedly decreased in the DSS group. However, DSS-induced claudin-1 and ZO-1 downregulation was notably reversed by $200 \mathrm{mg} / \mathrm{kg}$ ASI (Fig. 5). These data demonstrated that ASI increased the expression levels of TJ proteins in colonic tissues.

\section{Discussion}

Several compounds isolated from traditional Chinese medicines have been identified to have therapeutic effects in UC $(2,28)$. However, whether ASI has anti-UC activity remains unknown. Studies addressing this question has been very limited to date, to the best of our knowledge. The results of the present study indicated that ASI reduced the production of inflammatory cytokines and proteins in LPS-stimulated CCD-18Co cells in vitro. In addition, ASI alleviated the inflammatory response in the colon in DSS-induced UC in vivo. These data highlighted that ASI may exert its anti-inflammatory action on experimental UC in vitro and in vivo. To the best of our knowledge, this is the first study of the effects of ASI on experimental UC in vitro and in vivo.

The novel data from the present study is that ASI reversed LPS-induced TNF- $\alpha$, IL- $\beta$ and IL- 6 inflammatory cytokine increases via the inhibition of the NF- $\kappa \mathrm{B}$ pathway in vitro. TNF- $\alpha$, IL- $1 \beta$ and IL- 6 are pro-inflammatory cytokines associated with both colorectal and colitis-associated disease (29). Once these pro-inflammatory cell factors are delivered from immune cells, they increase inflammation and lead to mucosal tissue injury (26). Biologically, the inflammatory process is strongly mediated by NF- $\kappa \mathrm{B}$, a critical transcription factor for various pro-inflammatory cytokines including TNF- $\alpha$, IL- $\beta$ and IL- 6 . NF- $\kappa B$ serves as an inactive heterotrimer, including p50, p65 and I $\kappa$ B subunits in the cytoplasm (30). A recent study indicated that ASI ameliorated LPS-induced 

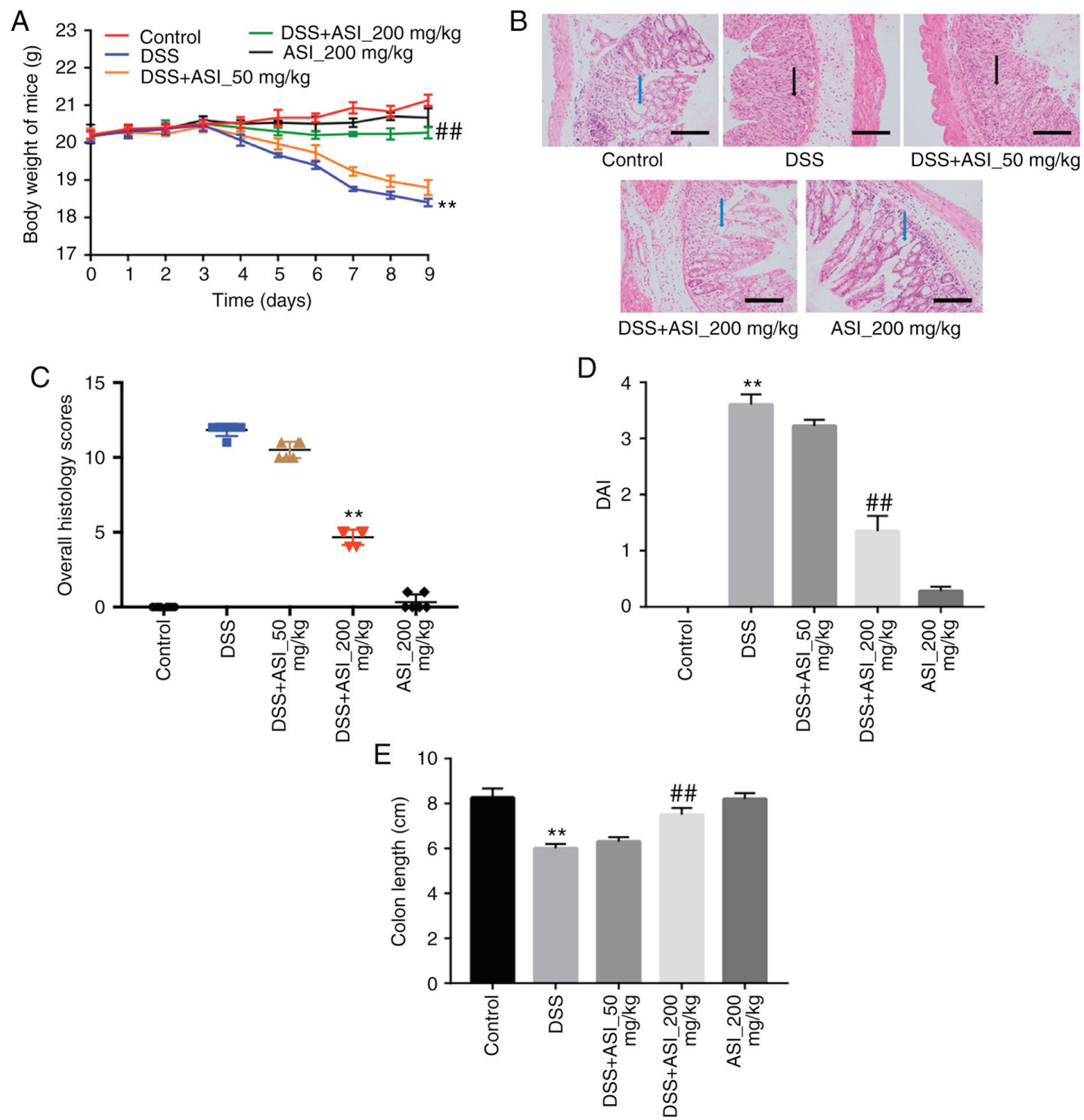

Figure 3. ASI attenuates ulcerative colitis in mice induced by DSS in vivo. (A) Bodyweight change of mice in each group. (B) The colon tissues in mice from each experimental group were processed for histological evaluation. Blue arrows represent crypt cells, black arrows represent polymorphonuclear neutrophils. Scale bars $=100 \mu \mathrm{m}$. Magnification, $\mathrm{x} 400$. (C) Histology damage scores for colon tissues. (D) DAI of mice in each group was evaluated. (E) The colon lengths of mice in each group were measured. ${ }^{* *} \mathrm{P}<0.01$ vs. the control group. ${ }^{\#} \mathrm{P}<0.01$ vs. the DSS-treated group. ASI, astragaloside IV; DSS, dextran sulfate sodium; DAI, disease activity index.

neuroinflammation in mice by decreasing the levels of TNF- $\alpha$ and IL-1 $\beta$ (31). Xu et al (32) identified that ASI suppressed synoviocytes and collagen-induced arthritis by inhibiting the levels of TNF- $\alpha$, IL- 6 and IL-1 $\beta$. Collectively, these results demonstrate that ASI has a potent inhibitory effect on $\mathrm{NF}-\kappa \mathrm{B}-$ mediated cytokine expression.

The DSS-induced UC model may be used to examine the pathogenesis of UC in humans, due to its similarity with the natural history of the disease in humans (1). It is well-known that weight loss and DAI in mice are commonly used to evaluate severity of UC (1). The results of the present study demonstrated that ASI $(200 \mathrm{mg} / \mathrm{kg})$ markedly reversed DSS-induced colonic shortening and weight loss in UC model mice, which indicated an attenuation of clinical UC symptoms. Consistently, Zhang et al (1) indicated that Zanthoxylum bungeanum pericarp extract alleviates DSS-induced colonic shortening and weight loss in an experimental colitis model in vivo. These results demonstrated that ASI (200 mg/kg) significantly alleviated the inflammatory response in the colon in the DSS-induced UC mouse model.

Importantly, MPO released from inflamed enteric mucosa and bowel tissues also serves a vital role in local bowel injury (33). In addition, NO is one of the primary sources of free radicals, which may additionally cause oxidative damage and intestinal lesions (2). The present study also examined the effect of ASI on MPO and NO production in colonic tissues. ASI $(200 \mathrm{mg} / \mathrm{kg})$ significantly inhibited the generation of MPO compared with the model group. Meanwhile, ASI (200 mg/kg) markedly decreased NO production in the colon tissues of UC mice compared with the model group. Kannan et al (34) demonstrated that Bauhinia tomentosa attenuated acetic acid-induced UC by regulating the levels of MPO and NO. Han et al (35) also identified that Xiexin decoction improves the inflammation associated with colitis and inhibits colonic inflammatory 

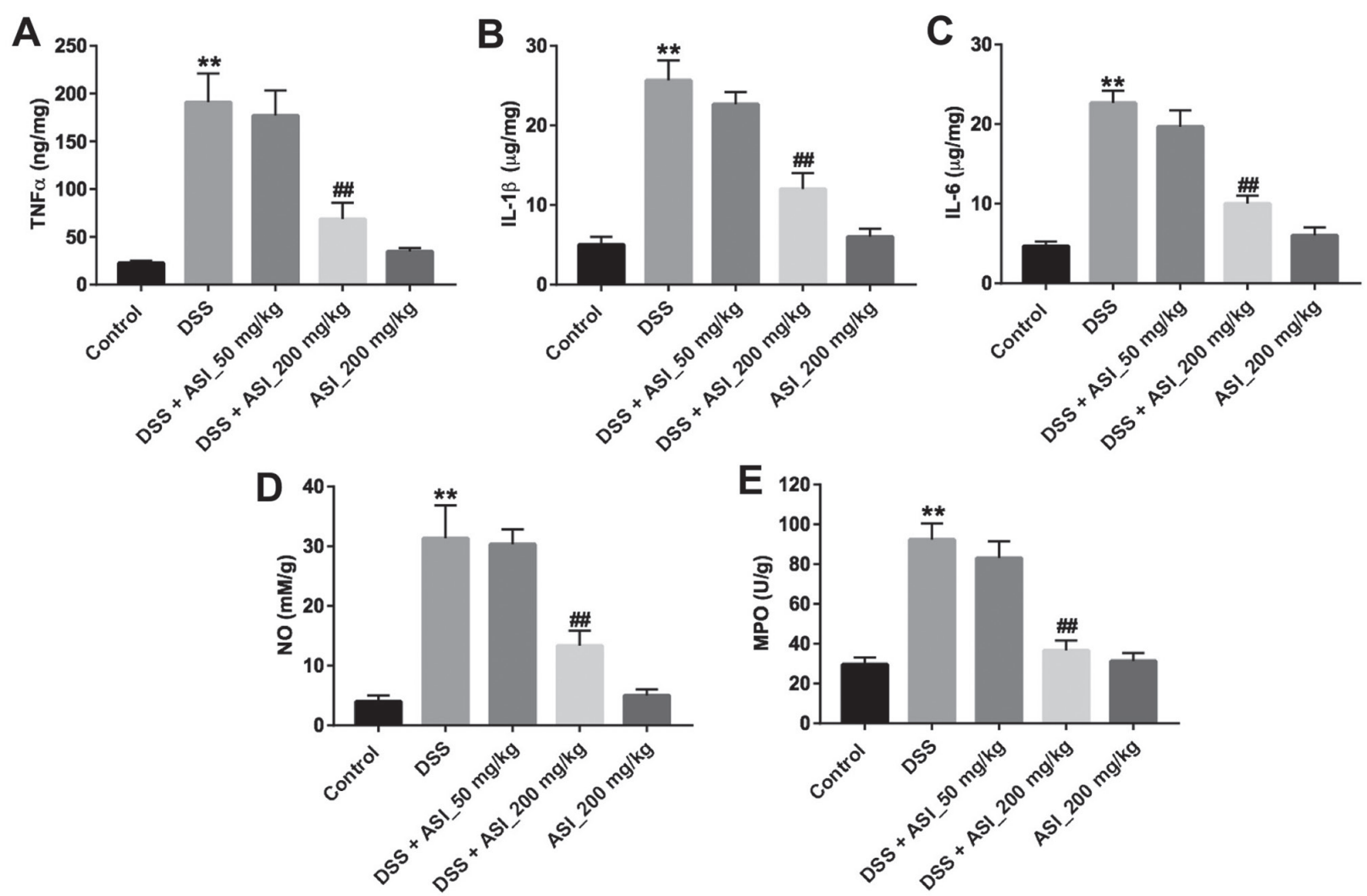

Figure 4. ASI alleviates inflammatory responses of colon in DSS-induced ulcerative colitis mice. The levels of (A) TNF- $\alpha$, (B) IL-1 $\beta$, (C) IL-6, (D) NO and (E) MPO in colon tissues were measured by ELISA. ${ }^{* *} \mathrm{P}<0.01$ vs. the control group. ${ }^{\# \#} \mathrm{P}<0.01$ vs. the DSS-treated group. ASI, astragaloside IV; DSS, dextran sulfate sodium; TNF- $\alpha$, tumor necrosis factor- $\alpha$; IL, interleukin; NO, nitric oxide; MPO, myeloperoxidase.
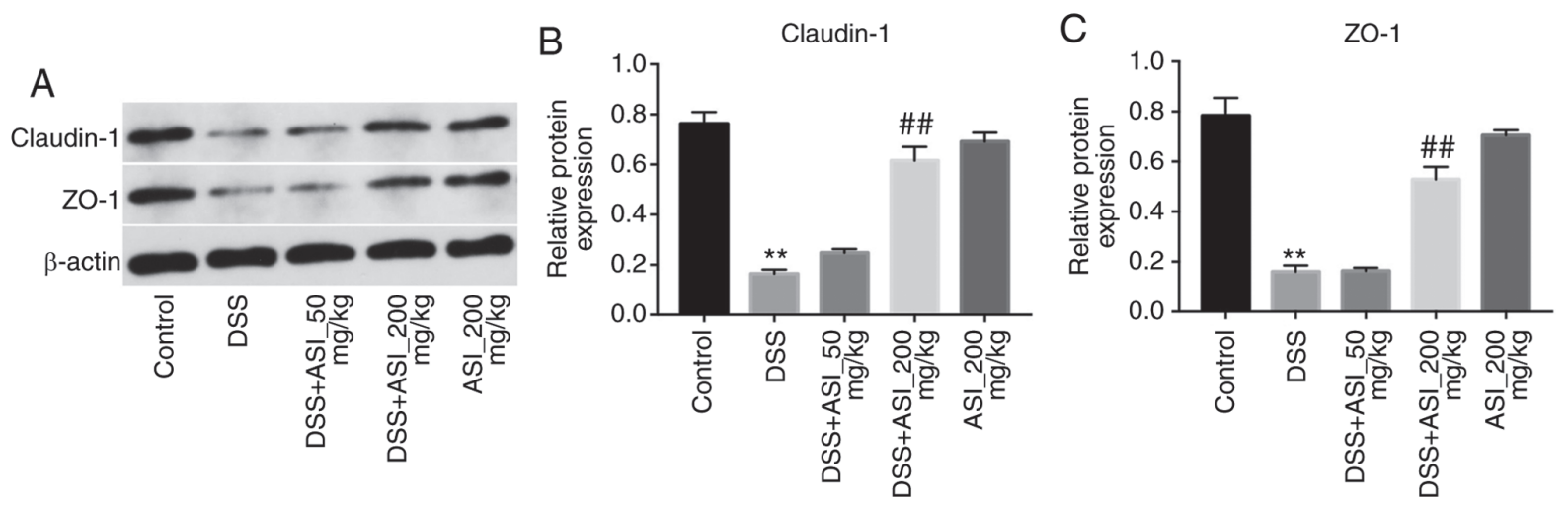

Figure 5. ASI increases the expression of tight junction proteins in colon tissues. (A) The expression of claudin-1 and ZO-1 in colon tissues of mice in each group were investigated via western blot analysis. (B) The relative level of claudin-1 was quantified via normalization to $\beta$-actin. (C) The relative level of ZO-1 was quantified via normalization to $\beta$-actin. ${ }^{* *} \mathrm{P}<0.01$ vs. the control group. ${ }^{\# \#} \mathrm{P}<0.01$ vs. the DSS-treated group. ASI, astragaloside IV; ZO-1, tight junction protein ZO-1; DSS, dextran sulfate sodium.

damage by decreasing the level of MPO in UC rats. The data from the present study were consistent with these results.

Clinical data has indicated that pro-inflammatory cytokines, including IL-6, TNF- $\alpha$ and IL-1 $\beta$, also perform important roles in UC pathogenesis in vivo (36). Jiang et al (19) demonstrated that ASI alleviated 2,4,6-trinitrobenzene sulfonic acid-induced inflammation in inflammatory bowel disease. Based on these aforementioned results, the present study investigated whether ASI had an effect on these pro-inflammatory factors during UC. The present results indicated that ASI $(200 \mathrm{mg} / \mathrm{kg})$ significantly downregulated the production of TNF- $\alpha$, IL- $1 \beta$ and
IL-6 in colon tissues compared with the model group. These data suggested that ASI may alleviate inflammation of the colonic tissues through downregulation of pro-inflammatory cytokines.

TJ proteins, including ZO-1 and claudin-1, form physiologically active regions in colonic tissues that may become disorganized in UC (27). For example, a previous study revealed that levels of key TJ proteins ZO-1 and claudin-1 were decreased in UC experimental models (37). Consistently, the present results indicated that ASI upregulated the expression of claudin-1 and ZO-1 proteins in DSS-treated mice. 
The results of the present study were limited in that the therapeutic strategy was not adjusted according to the disease stage of UC in the animal studies. Future studies will be required to explore the effect of ASI on UC in different disease stages.

In summary, ASI was effective in ameliorating experimental UC in vitro and in vivo via the inhibition of inflammatory molecule production and the downregulation of $\mathrm{NF}-\kappa \mathrm{B}$ signaling. These results suggest that ASI may serve as a potential therapeutic agent for the treatment of UC.

\section{Acknowledgements}

Not applicable.

\section{Funding}

No funding was received.

\section{Availability of data and materials}

The datasets used and/or analyzed during the current study are available from the corresponding author on reasonable request.

\section{Authors' contributions}

SW and ZC analyzed and interpreted the experimental data, and were major contributors to the development of the first draft of the present manuscript. ZC reviewed and approved the final draft of the manuscript prior to submission.

\section{Ethics approval and consent to participate}

Ethics approval for the present study was approved by the Ethical Committee of Suzhou Integrated Traditional Chinese and Western Medicine Hospital (Suzhou, China).

\section{Patient consent for publication}

Not applicable.

\section{Conflicts of interest}

The authors declare that they have no competing interests.

\section{References}

1. Zhang Z, Liu J, Shen P, Cao Y, Lu X, Gao X, Fu Y, Liu B and Zhang N: Zanthoxylum bungeanum pericarp extract prevents dextran sulfate sodium-induced experimental colitis in mice via the regulation of TLR4 and TLR4-related signaling pathways. Int Immunopharmacol 41: 127-135, 2016.

2. Zhang H, Deng A, Zhang Z, Yu Z, Liu Y, Peng S, Wu L, Qin H and Wang W: The protective effect of epicatechin on experimental ulcerative colitis in mice is mediated by increasing antioxidation and by the inhibition of $\mathrm{NF}-\kappa \mathrm{B}$ pathway. Pharmacol Rep 68: 514-520, 2016.

3. Harris KG and Chang EB: The intestinal microbiota in the pathogenesis of inflammatory bowel diseases: New insights into complex disease. Clin Sci (Lond) 132: 2013-2028, 2018.

4. Yadav V, Varum F, Bravo R, Furrer E, Bojic D and Basit AW: Inflammatory bowel disease: Exploring gut pathophysiology for novel therapeutic targets. Transl Res 176: 38-68, 2016.
5. Ordás I, Eckmann L, Talamini M, Baumgart DC and Sandborn WJ: Ulcerative colitis. Lancet 380: 1606-1619, 2012.

6. Thia KT, Loftus EV Jr, Sandborn WJ and Yang SK: An update on the epidemiology of inflammatory bowel disease in Asia. Am J Gastroenterol 103: 3167-3182, 2008.

7. Peyrin-Biroulet L, Deltenre P, Ardizzone S, D'Haens G, Hanauer SB, Herfarth H, Lémann $M$ and Colombel JF: Azathioprine and 6-mercaptopurine for the prevention of postoperative recurrence in Crohn's disease: A meta-analysis. Am J Gastroenterol 104: 2089-2096, 2009.

8. Kemp R, Dunn E and Schultz M: Immunomodulators in inflammatory bowel disease: An emerging role for biologic agents. BioDrugs 27: 585-590, 2013.

9. Atanasov AG, Waltenberger B, Pferschy-Wenzig EM, Linder T, Wawrosch C, Uhrin P, Temml V, Wang L, Schwaiger S, Heiss EH, et al: Discovery and resupply of pharmacologically active plant-derived natural products: A review. Biotechnol Adv 33: 1582-1614, 2015.

10. Maria-Ferreira D, Nascimento AM, Cipriani TR, Santana-Filho AP, Watanabe PDS, Sant Ana DMG, Luciano FB, Bocate KCP, van den Wijngaard RM, Werner MFP and Baggio $\mathrm{CH}$ : Rhamnogalacturonan, a chemically-defined polysaccharide, improves intestinal barrier function in DSS-induced colitis in mice and human Caco- 2 cells. Sci Rep 8: 12261, 2018.

11. Sun Y, Zhao Y, Yao J, Zhao L, Wu Z, Wang Y, Pan D, Miao H, Guo Q and Lu N: Wogonoside protects against dextran sulfate sodium-induced experimental colitis in mice by inhibiting NF- $\kappa \mathrm{B}$ and NLRP3 inflammasome activation. Biochem Pharmacol 94: 142-154, 2015

12. Yang L, Xing F, Han X, Li Q, Wu H, Shi H, Wang Z, Huang F and $\mathrm{Wu} \mathrm{X}$ : Astragaloside IV regulates differentiation and induces apoptosis of activated CD4+ T cells in the pathogenesis of experimental autoimmune encephalomyelitis. Toxicol Appl Pharmacol 362: 105-115, 2019.

13. Xu N, Kan P, Yao X, Yang P, Wang J, Xiang L and Zhu Y: Astragaloside IV reversed the autophagy and oxidative stress induced by the intestinal microbiota of AIS in mice. J Microbiol 56: 838-846, 2018.

14. Dai PC, Liu DL, Zhang L, Ye J, Wang Q, Zhang HW, Lin XH and Lai GX: Astragaloside IV sensitizes non-small cell lung cancer cells to gefitinib potentially via regulation of SIRT6. Tumour Biol 39: 1010428317697555, 2017.

15. Dong Z, Zhou J, Zhang Y, Chen Y, Yang Z, Huang G, Chen Y, Yuan Z, Peng Y and Cao T: Astragaloside-IV alleviates heat-induced inflammation by inhibiting endoplasmic reticulum stress and autophagy. Cell Physiol Biochem 42: 824-837, 2017.

16. Zhou X, Sun X, Gong X, Yang Y, Chen C, Shan G and Yao Q: Astragaloside IV from Astragalus membranaceus ameliorates renal interstitial fibrosis by inhibiting inflammation via TLR4/NF-KB in vivo and in vitro. Int Immunopharmacol 42: 18-24, 2017

17. Kang JH, Choi S, Jang JE, Ramalingam P, Ko YT, Kim SY and Oh SH: Wasabia japonica is a potential functional food to prevent colitis via inhibiting the NF- $\kappa \mathrm{B}$ signaling pathway. Food Funct 8 : 2865-2874, 2017.

18. Zhao P, Wang Y, Zeng S, Lu J, Jiang TM and Li YM: Protective effect of astragaloside IV on lipopolysaccharide-induced cardiac dysfunction via downregulation of inflammatory signaling in mice. Immunopharmacol Immunotoxicol 37 : 428-433, 2015

19. Jiang XG, Sun K, Liu YY, Yan L, Wang MX, Fan JY, Mu HN, Li C, Chen YY, Wang CS and Han JY: Astragaloside IV ameliorates 2,4,6-trinitrobenzene sulfonic acid (TNBS)-induced colitis implicating regulation of energy metabolism. Sci Rep 7: 41832, 2017.

20. He X, Zheng Z, Yang X, Lu Y, Chen N and Chen W: Tetramethylpyrazine attenuates PPAR- $\gamma$ antagonist-deteriorated oxazolone-induced colitis in mice. Mol Med Rep 5: 645-650, 2012.

21. National Research Council (US) Committee for the Update of the Guide for the Care and Use of Laboratory Animals: Guide for the Care and Use of Laboratory Animals. 8th edition. National Academies Press (US), Washington, DC, 2011.

22. Zhou Q, Gong X, Kuang G, Jiang R, Xie T, Tie H, Chen X, Li K, Wan $\mathrm{J}$ and Wang B: Ferulic acid protected from kidney ischemia reperfusion injury in mice: Possible mechanism through increasing adenosine generation via HIF-1a. Inflammation 41: 2068-2078, 2018. 
23. Zheng B, van Bergenhenegouwen J, Overbeek S, van de Kant HJ, Garssen J, Folkerts G, Vos P, Morgan ME and Kraneveld AD: Bifidobacterium breve attenuates murine dextran sodium sulfate-induced colitis and increases regulatory $\mathrm{T}$ cell responses. PLoS One 9: e95441, 2014

24. Araki T, Hashimoto K, Okita Y, Fujikawa H, Kondo S, Kobayashi M, Ohi M, Toiyama Y, Inoue Y, Uchida K, et al: Colonic histological criteria predict development of pouchitis after Ileal Pouch: Anal anastomosis for patients with ulcerative colitis. Dig Surg 35: 138-143, 2018.

25. Mao S, Yang G, Li W, Zhang J, Liang H, Li J and Zhang M: Gastroprotective effects of Astragaloside IV against acute gastric lesion in rats. PLoS One 11: e0148146, 2016.

26. Ge H, Tang H, Liang Y, Wu J, Yang Q, Zeng L and Ma Z: Rhein attenuates inflammation through inhibition of NF- $\mathrm{BB}$ and NALP3 inflammasome in vivo and in vitro. Drug Des Devel Ther 11: 1663-1671, 2017

27. Kim Y, Wu AG, Jaja-Chimedza A, Graf BL, Waterman C, Verzi MP and Raskin I: Isothiocyanate-enriched moringa seed extract alleviates ulcerative colitis symptoms in mice. PLoS One 12: e0184709, 2017.

28. Qu C, Yuan ZW, Yu XT, Huang YF, Yang GH, Chen JN, Lai XP, Su ZR, Zeng HF, Xie Y and Zhang XJ: Patchouli alcohol ameliorates dextran sodium sulfate-induced experimental colitis and suppresses tryptophan catabolism. Pharmacol Res 121: 70-82, 2017.

29. Venancio VP, Cipriano PA, Kim H, Antunes LM, Talcott ST and Mertens-Talcott SU: Cocoplum (Chrysobalanus icaco L.) anthocyanins exert anti-inflammatory activity in human colon cancer and non-malignant colon cells. Food Funct 8: 307-314, 2017.

30. Buss H, Handschick K, Jurrmann N, Pekkonen P, Beuerlein K, Muller H, Wait R, Saklatvala J, Ojala PM, Schmitz ML, et al: Cyclin-dependent kinase 6 phosphorylates NF-кB P65 at serine 536 and contributes to the regulation of inflammatory gene expression. PLoS One 7: e51847, 2012.
31. Song MT, Ruan J, Zhang RY, Deng J, Ma ZQ and Ma SP: Astragaloside IV ameliorates neuroinflammation-induced depressive-like behaviors in mice via the PPAR $\gamma / \mathrm{NF}-\kappa \mathrm{B} / \mathrm{NLRP} 3$ inflammasome axis. Acta Pharmacol Sin 39: 1559-1570, 2018.

32. Xu H, Wang CY, Zhang HN, Lv CY and Wang YZ: Astragaloside IV suppresses inflammatory mediator production in synoviocytes and collagen-induced arthritic rats. Mol Med Rep 13: 3289-3296, 2016.

33. Chiu CT, Kuo SN, Hung SW and Yang CY: Combined treatment with hyaluronic acid and mesalamine protects rats from inflammatory bowel disease induced by intracolonic administration of trinitrobenzenesulfonic acid. Molecules 22: pii: E904, 2017.

34. Kannan N and Guruvayoorappan C: Protective effect of Bauhinia tomentosa on acetic acid induced ulcerative colitis by regulating antioxidant and inflammatory mediators. Int Immunopharmacol 16: 57-66, 2013.

35. Han XH, Zhong J, Guo JY, Shi R, Wang XH, Wang CH, Wang K, Du GL, Shen YH and Ma YM: Relationships between pharmacokinetics and efficacy of Xie-xin decoction in rats with experimental ulcerative colitis. J Ethnopharmacol 148: 182-189, 2013.

36. Neurath MF: Cytokines in inflammatory bowel disease. Nat Rev Immunol 14: 329-342, 2014.

37. Li Q, Zhang Q, Zhang M, Wang C, Zhu Z, Li N and Li J: Effect of n-3 polyunsaturated fatty acids on membrane microdomain localization of tight junction proteins in experimental colitis. FEBS J 275: 411-420, 2008

This work is licensed under a Creative Commons Attribution-NonCommercial-NoDerivatives 4.0 International (CC BY-NC-ND 4.0) License. 